\title{
A Precision Microbiome Approach Using Sucrose for Selective Augmentation of Staphylococcus epidermidis Fermentation against Propionibacterium acnes
}

\author{
Yanhan Wang ${ }^{1}$, Ming-Shan Kao ${ }^{2}$, Jinghua Yu ${ }^{3}$, Stephen Huang ${ }^{4}$, Shinta Marito ${ }^{2}$, \\ Richard L. Gallo ${ }^{1}$ and Chun-Ming Huang ${ }^{1,5, *}$ \\ 1 Department of Dermatology, School of Medicine, University of California, San Diego, CA 92093, USA; \\ yanhanw@gmail.com (Y.W.); rgallo@ucsd.edu (R.L.G.) \\ 2 Department of Biomedical Sciences and Engineering, National Central University, Taoyuan 320009, Taiwan; \\ s36424592@yahoo.com.tw (M.-S.K.); shintasimbolon53@yahoo.com (S.M.) \\ 3 NMR and Crystallography Facilities, Sanford-Burnham Institute for Medical Research, La Jolla, CA 92037, \\ USA; jinghua@sbpdiscovery.org \\ 4 Surface Bioadvances Inc., San Diego, CA 92121, USA; rhuang53@gmail.com \\ 5 Moores Cancer Center, University of California, San Diego, CA 92103, USA \\ * Correspondence: chunming@ucsd.edu; Tel.: +1-858-822-4627; Fax: +1-858-642-1435 \\ Academic Editor: Chris Jackson \\ Received: 5 August 2016; Accepted: 31 October 2016; Published: 9 November 2016
}

\begin{abstract}
Acne dysbiosis happens when there is a microbial imbalance of the over-growth of Propionibacterium acnes (P. acnes) in the acne microbiome. In our previous study, we demonstrated that Staphylococcus epidermidis (S. epidermidis, a probiotic skin bacterium) can exploit glycerol fermentation to produce short-chain fatty acids (SCFAs) which have antimicrobial activities to suppress the growth of $P$. acnes. Unlike glycerol, sucrose is chosen here as a selective fermentation initiator (SFI) that can specifically intensify the fermentation activity of $S$. epidermidis, but not $P$. acnes. A co-culture of $P$. acnes and fermenting $S$. epidermidis in the presence of sucrose significantly led to a reduction in the growth of $P$. acnes. The reduction was abolished when $P$. acnes was co-cultured with non-fermenting S. epidermidis. Results from nuclear magnetic resonance (NMR) analysis revealed four SCFAs (acetic acid, butyric acid, lactic acid, and succinic acid) were detectable in the media of S. epidermidis sucrose fermentation. To validate the interference of $S$. epidermidis sucrose fermentation with P. acnes, mouse ears were injected with both $P$. acnes and S. epidermidis plus sucrose or phosphate buffered saline (PBS). The level of macrophage-inflammatory protein-2 (MIP-2) and the number of P. acnes in ears injected with two bacteria plus sucrose were considerably lower than those in ears injected with two bacteria plus PBS. Our results demonstrate a precision microbiome approach by using sucrose as a SFI for S. epidermidis, holding future potential as a novel modality to equilibrate dysbiotic acne.
\end{abstract}

Keywords: acne vulgaris; microbiome; P. acnes; S. epidermidis; skin; sucrose

\section{Introduction}

Microbial imbalance in the human skin microbiome [1,2] has been termed "skin dysbiosis" [3]. Here we refer to the precision microbiome as an approach for normalization of dysbiotic microbiome by selectively targeting specific microbes. Propionibacterium acnes (P. acnes) and Staphylococcus epidermidis (S. epidermidis) are two major bacterial inhabitants of lesions in acne vulgaris [2]. Although both P. acnes and S. epidermidis are human skin commensals, the over-growth of $P$. acnes has been recognized for its association with the progression of acne vulgaris [1,3]. The biological role of S. epidermidis within acne lesions remains mysterious. During the development of acne vulgaris, S. epidermidis may irregularly migrate from the outer surface of the skin into the hair follicle, where opportunistic strains of P. acnes 
exclusively habitate. A closed comedone (or a deep-seated abscess in an open comedone) creates an anaerobic microenvironment which may facilitate bacterial fermentation in an acne lesion. Results in our publication have demonstrated that $S$. epidermidis can ferment glycerol and create inhibition zones to repel a colony of over-grown P. acnes [4]. Both topical and intralesional application of a short-chain fatty acid (SCFA) produced by S. epidermidis glycerol fermentation into P. acnes-induced lesions markedly suppressed the bacterial colonization and inflammation in mice [4]. Although S. epidermidis is not well-defined as a probiotic bacterium, our previous results suggest that $S$. epidermidis can exploit glycerol fermentation against $P$. acnes.

Both S. epidermidis and P. acnes can fermentatively metabolize glycerol to produce SCFAs $[4,5]$. Although the oppositional relationship between S. epidermidis and P. acnes in human skin has not yet been clinically proven, we conjecture that, during the development of acne vulgaris, S. epidermidis and P. acnes may use the glycerol as a shared carbon source and produce different SCFAs as antimicrobial agents to compete against each other within an acne lesion. The acne vulgaris develops and persists when $P$. acnes becomes dominant during the bacterial interference. Our approach to treating acne vulgaris is to deter the over-growth of $P$. acnes by enhancing the fermentation activities of S. epidermidis by using a specific sugar substrate as a selective fermentation initiator (SFI).

It has been reported that different bacterial species express distinct enzymes that ferment specific sugar substrates [6,7]. Many bacteria use glucose, because they possess the enzymes required for the degradation and oxidation of this sugar. Fewer bacteria are able to use complex carbohydrates like disaccharides (lactose or sucrose) or polysaccharides (starch); these bacteria produce enzymes to hydrolyse glycosidic bonds of disaccharides. The monosaccharides from degraded disaccharides become new substrates for bacterial fermentation. S. epidermidis, but not Staphylococcus aureus (S. aureus), can ferment mannose and galactose [6,7]. Our previous results showed that both S. epidermidis and P. acnes, but not S. aureus (USA300), can fermentatively metabolize glycerol [8]. Although $S$. aureus bacteria utilize several carbohydrates as substrates for fermentation, they cannot ferment ducitol (also called galactitol, a sugar alcohol) or saccharic acid [7]. Unlike glucose and glycerol, which are endogenous metabolites in human skin, sucrose $\left(\mathrm{C}_{12} \mathrm{H}_{22} \mathrm{O}_{11}\right)$-a disaccharide combination of glucose and fructose sugars-is found naturally in many fruits and vegetables. It has been documented that sucrose is nonfermentable by P. acnes, and it has been used to reduce water activity and hence bacterial colonization of wounds [9].

Antibiotics without bacterial specificity for acne treatment may destroy the fermenting bacteria that help rein in the over-growth of $P$. acnes and maintain homeostasis of the acne microbiome. At dermatology clinics, acne cyst injections by direct injection of corticosteroids into the nodule can swiftly reduce redness and inflammation and help to immediately relieve the threat of scarring. However, this injection can cause local side effects, including pigmentary changes and atrophy [10]. Benzoyl peroxide- a topical medication for acne vulgaris—can cause severe skin irritation. Isotretinoin is a potent anti-acne agent derived from vitamin A [11]. However, it is strictly regulated because of its known adverse effects on birth defects. However, it is strictly regulated due to the induction of unwanted side effects. None of the above treatments selectively improve the fermentation activity of commensal skin bacteria that may have a lower risk of developing side effects and resistant $P$. acnes. Unlike vaccines, the action of probiotics does not directly require host immune stimulation, and may have little or no disruption to other commensal bacteria. However, live S. epidermidis as probiotics, when added exogenously into the open lesion/comedone of acne vulgaris, may be not a viable option in patients with significant underlying health issues, such as surgical intervention and immune suppression. In this study, we employ a precision microbiome approach by selective augmentation of the fermentation activities of S. epidermidis against $P$. acnes. The development of sucrose as a targeted intervention specifically for S. epidermidis fermentation may be relatively safe when it is employed to rebalance dysbiotic acne. 


\section{Results}

\subsection{Sucrose Selectively Triggered S. epidermidis, but Not P. acnes, to Undergo Fermentation}

To examine the sucrose fermentation activities of $S$. epidermidis and P. acnes, bacteria were incubated in rich medium under anaerobic conditions in the presence of $20 \mathrm{~g} / \mathrm{L}$ sucrose. Rich media plus either sucrose or bacteria were used as controls. To monitor the fermentation process, phenol red (a fermentation indicator) was added into the culture to assess SCFA production as a result of sucrose fermentation. Only media in the culture of S. epidermidis (ATCC 12228), but not P. acnes (ATCC 6919), with sucrose turned yellow (more acidic) after six days of incubation (Figure 1), indicating that sucrose can selectively trigger S. epidermidis to undergo fermentation.

A

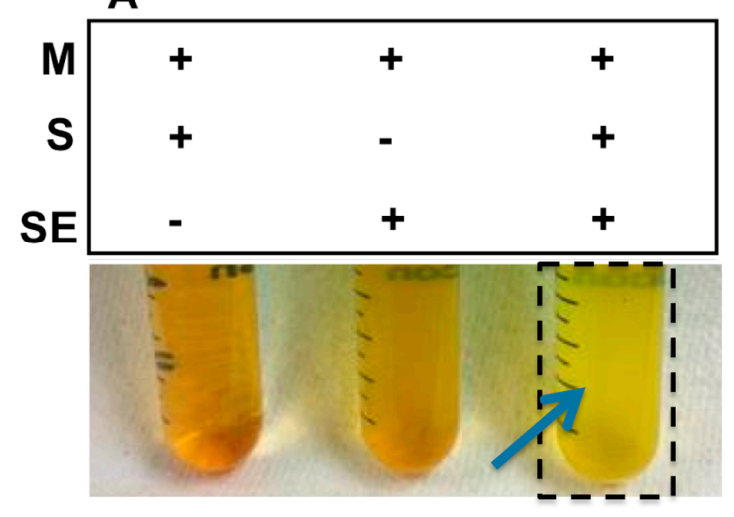

B

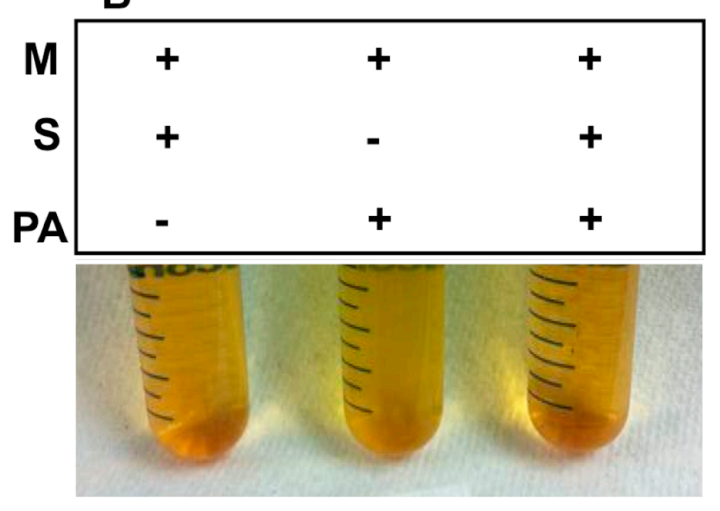

Figure 1. Sucrose as a selective carbon source for Staphylococcus epidermidis (S. epidermidis) fermentation. (A) S. epidermidis (SE) or (B) Propionibacterium acnes (P. acnes, PA) $\left(10^{5} \mathrm{CFU} / \mathrm{mL}\right)$ was incubated in rich media (M) with or without $20 \mathrm{~g} / \mathrm{L}$ sucrose (S) for six days. Rich media plus sucrose without bacteria was included as a control. S. epidermidis, but not P. acnes, fermented sucrose. A color change to yellow in the media (marked in a black frame and blue arrow) indicates that sucrose fermentation of S. epidermidis has occurred. Representative data from three independent experiments are shown.

\subsection{The Sucrose Fermentation of S. epidermidis Is Essential for Inhibition of P. acnes Growth}

To examine whether the sucrose fermentation of $S$. epidermidis hindered the growth of P. acnes, S. epidermidis and P. acnes were co-cultured in the presence or absence of sucrose. To establish a $P$. acnes-selective plate, media from the co-culture of $S$. epidermidis and P. acnes were spotted on a rich medium plate supplemented $10 \mu \mathrm{g} / \mathrm{mL}$ of furazolidone (Furoxone). We found that furazolidone at a concentration of $10 \mu \mathrm{g} / \mathrm{mL}$ can completely kill S. epidermidis without affecting the growth of $P$. acnes (data not shown). Three days after the co-culture of S. epidermidis and P. acnes with/without sucrose, media were spotted on a $P$. acnes-selective plate. After co-culture of $S$. epidermidis /P. acnes in the absence of sucrose, $P$. acnes formed $1.4 \pm 0.29 \times 10^{5}$ CFUs on a plate. However, when sucrose was present in the co-culture, an approximately one $\log _{10}$ reduction in the number of $P$. acnes colonies $\left(1.2 \pm 0.01 \times 10^{4} \mathrm{CFUs}\right)$ was observed (Figure 2A). These findings suggest that S. epidermidis mediated sucrose fermentation to interfere with the growth of $P$. acnes.

To validate the essential role of $S$. epidermidis fermentation in the interference with the growth of $P$. acnes, we screened the fermentation activities of different $S$. epidermidis strains isolated from human fingertips. In the presence of sucrose, the media in the culture of a strain with a 16S rRNA sequence showing 99\% identity to that in S. epidermidis ATCC 12228 [12] remained orange-red after six days of incubation (Figure S1). We thus define this strain as a non-fermenting S. epidermidis. When a co-culture of non-fermenting $S$. epidermidis with $P$. acnes was conducted in the presence of sucrose, no reduction in the number of $P$. acnes colonies was found (Figure 2B), suggesting that fermentation is required for S. epidermidis to restrain the growth of P. acnes. 

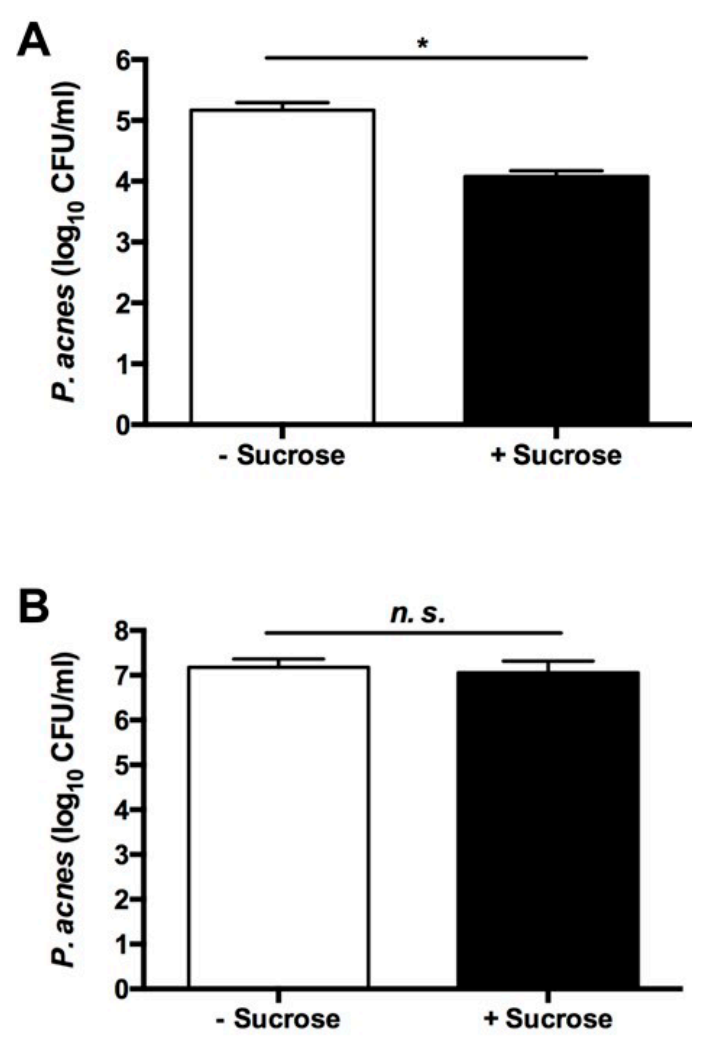

Figure 2. The essential role of sucrose fermentation of S. epidermidis in the inhibition of the growth of P. acnes. (A) Fermenting S. epidermidis (ATCC 12228) or (B) non-fermenting S. epidermidis (106 CFU) were co-incubated with P. acnes $\left(10^{6} \mathrm{CFU}\right)$ in $10 \mathrm{~mL}$ rich media with or without $20 \mathrm{~g} / \mathrm{L}$ sucrose for three days. After incubation, culture media containing bacteria were diluted 1:10-1:10 5 with phosphate buffered saline (PBS) and then spotted the dilution $(5 \mu \mathrm{L})$ onto P. acnes selective agar plates which contain rich media and $10 \mu \mathrm{g} / \mathrm{mL}$ of furazolidone. The number of $P$. acnes six days after incubation is expressed as $\log _{10} \mathrm{CFU} / \mathrm{mL}$. Data are the mean \pm standard deviation (SD) of three separate experiments. ${ }^{*} p<0.05$ (two-tailed $t$-tests). n.s. $=$ not significant.

\subsection{Identified SCFAs in Fermented Media of S. epidermidis}

To identify the SCFAs during fermentation, ${ }^{13} \mathrm{C}_{12}$-sucrose $(20 \mathrm{~g} / \mathrm{L})$ was added into the culture of S. epidermidis (ATCC 12228) under anaerobic conditions for six days. Supernatants of bacterial culture were mixed with $10 \%$ deuterium oxide $\left(\mathrm{D}_{2} \mathrm{O}\right)$ for one-dimensional (1-D) (data not shown) and two-dimensional (2-D) (Figure 3$){ }^{13} \mathrm{C}$ and ${ }^{1} \mathrm{H}$ nuclear magnetic resonance (NMR) analysis. Besides un-metabolized sucrose, four SCFAs (acetic, butyric, lactic, and succinic acids) were detected in the fermented media of S. epidermidis, demonstrating the bacterial capability of fermentatively metabolizing sucrose into SCFAs. These results demonstrate that S. epidermidis fermentatively metabolized ${ }^{13} \mathrm{C}_{12}$-sucrose into SCFAs. Results in our previous publication have demonstrated that SCFAs can suppress the growth of P. acnes ATCC 6919 [4]. To examine if SCFAs have the ability to kill $P$. acnes strains isolated from acne lesions, the minimum bactericidal concentration (MBC) value of acetic acid for a P. acnes strain isolated from acne lesion was determined (Figure S2). P. acnes was incubated with varying concentrations $(0,5,7.5,10,25$ and $50 \mathrm{mM})$ of acetic acid overnight at $37^{\circ} \mathrm{C}$. After incubation, bacteria diluted with PBS were spotted on an agar plate for counting the colony forming units (CFU). Acetic acid reduced the P. acnes growth by more than one $\log _{10}$ at a concentration greater than $7.5 \mathrm{mM}$, and completely killed P. acnes at a concentration higher than $25 \mathrm{mM}$. These results suggest that SCFAs produced by sucrose fermentation of S. epidermidis may be able to impede the growth of $P$. acnes in acne vulgaris. 


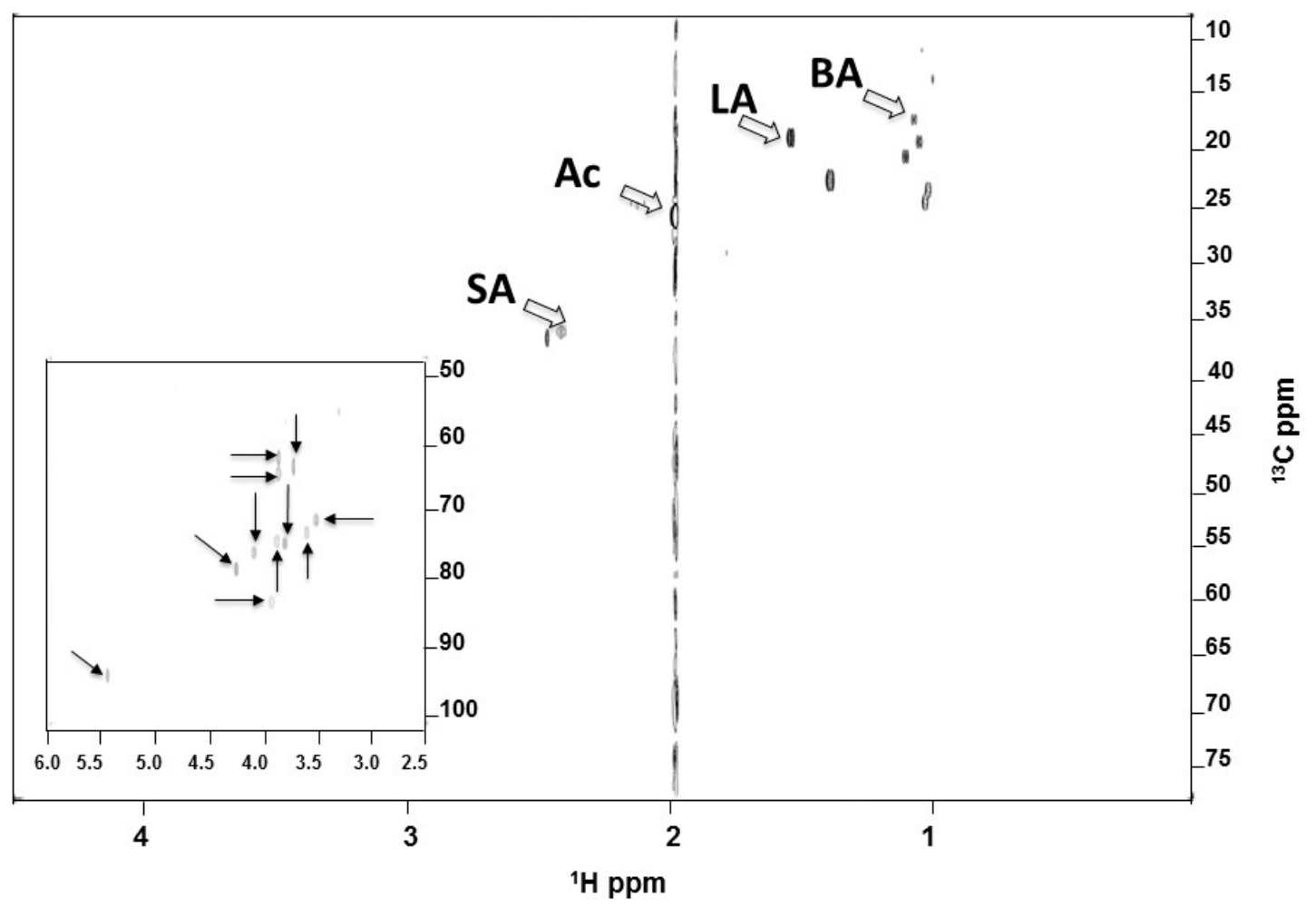

Figure 3. Short-chain fatty acid (SCFA) identification by NMR analysis. The media of S. epidermidis ${ }^{13} \mathrm{C}_{12}$-sucrose fermentation were centrifuged and passed through a $0.2 \mu \mathrm{m}$ filter. Supernatants were then mixed with $10 \% \mathrm{D}_{2} \mathrm{O}$ and analyzed by NMR spectrometers. A 2-D ${ }^{1} \mathrm{H}-{ }^{13} \mathrm{C}$ HSQC NMR spectrum $(600 \mathrm{MHz})$ was displayed. The un-metabolized ${ }^{13} \mathrm{C}_{12}$-sucrose (solid arrows) appears $2.5-6.0$ and 50-100 ppm in the ${ }^{1} \mathrm{H}$ - and ${ }^{13} \mathrm{C}-\mathrm{NMR}$ spectra, respectively. Besides sucrose, four SCFAs (acetic (Ac), butyric (BA), lactic (LA), and succinic acids (SA), open arrows) were detected in the ferments of $S$. epidermidis.

\subsection{S. epidermidis Sucrose Fermentation Abrogated P. acnes-Induced Inflammation and Bacteria Colonization} In Vivo

To test whether S. epidermidis can counteract $P$. acnes in the presence of sucrose, the ears of Institute for Cancer Research (ICR) mice were injected intradermally with P. acnes $\left(10^{7} \mathrm{CFU}\right)$ and S. epidermidis $\left(10^{7} \mathrm{CFU}\right)$ in the presence of sucrose $(20 \mathrm{~g} / \mathrm{L})$ or PBS for three days. As shown in Figure 4, compared to those in the mice injected with two bacteria and PBS, both ear redness and thickness in the mice injected with two bacteria and sucrose was significantly lower (Figure 4A,B). To determine whether S. epidermidis sucrose fermentation can ameliorate the production of P. acnes-induced pro-inflammatory cytokines, mouse ears were excised and homogenized three days after injection. The level of macrophage-inflammatory protein-2 (MIP-2) - a murine counterpart of human interleukin (IL) 8-was measured by an enzyme-linked immunosorbent assay (ELISA). MIP-2 production in the ear injected with two bacteria and sucrose was approximately $75 \%$ (solid bar; $0.84 \pm 0.17 \times 10^{4} \mathrm{pg} / \mathrm{mL}$ ) less than that detected in the ear injected with two bacteria and PBS (open bar; $3.26 \pm 0.51 \times 10^{4} \mathrm{pg} / \mathrm{mL}$ ) (Figure 4C).

To explore if S. epidermidis sucrose fermentation can hinder the growth of P. acnes, the ears of mice injected with two bacteria along with sucrose or phosphate buffered saline (PBS) were excised and homogenized. As shown in Figure 4D, the number of P. acnes recovered from mouse ears administered with two bacteria plus sucrose (solid bar; $6.0 \pm 0.07 \times 10^{4} \mathrm{CFUs}$ ) was much lower than that recovered from ears administered with two bacteria plus PBS (open bar; $12.9 \pm 1.1 \times 10^{5} \mathrm{CFUs}$ ), suggesting the suppression of $P$. acnes growth by $S$. epidermidis sucrose fermentation. 


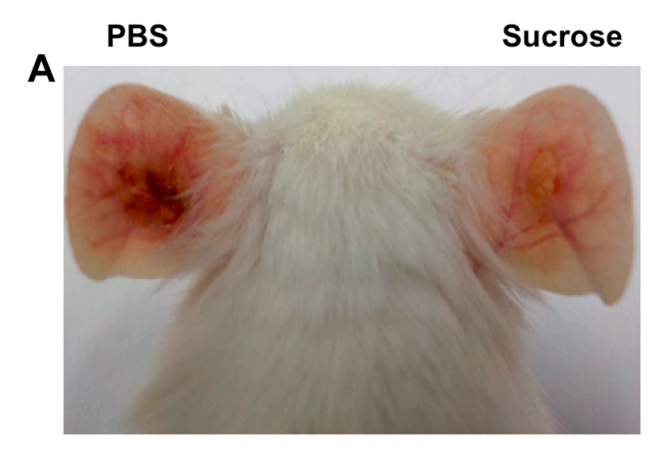

D
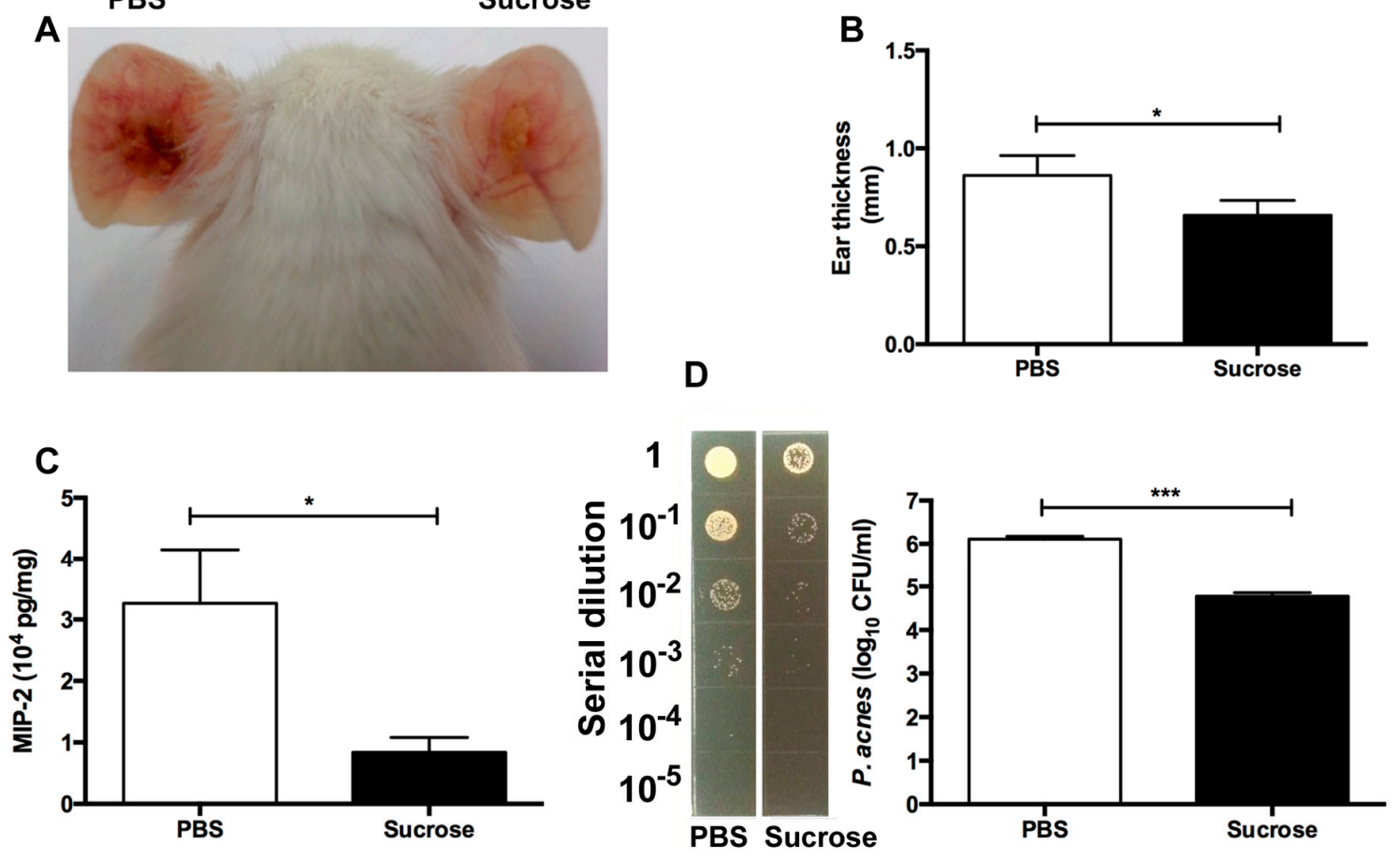

Figure 4. In Vivo reduction of P. acnes colonization and inflammation by S. epidermidis sucrose fermentation. The ears of Institute for Cancer Research (ICR) mice were injected intradermally with P. acnes (ATCC 6919) (10 7 CFU) and S. epidermidis (ATCC 12228) (10 7 CFU) with sucrose (20 g/L in $10 \mu \mathrm{L}$ PBS) or $10 \mu \mathrm{L}$ PBS. (A) A photo of ear inflammation was taken three days after injection; (B) The ear thickness (mm); (C) The levels of macrophage-inflammatory protein-2 (MIP-2) cytokines in the ears injected with bacteria in the presence of sucrose or PBS was measured by an enzyme-linked immunosorbent assay (ELISA) kit; (D) The CFUs in the ears injected with bacteria in the presence of sucrose or PBS were enumerated by plating serial dilutions $\left(1: 10-1: 10^{5}\right)$ of the homogenate on an agar plate. Three days after injection, $p$-values were evaluated using two-tailed $t$-tests. Data are the means of three separate experiments using four mice per group. ${ }^{* *} p<0.001{ }^{*} p<0.05$.

\section{Discussion}

Sugars hold inherent antibacterial properties, some of which are due to high osmotic pressure and low water activity, which is inhibitory to the growth of bacteria [13]. It has been reported that the inhibitory effect of sucrose against $S$. aureus was primarily attributed to its water activity-lowering ability [14]. To examine the possible antibacterial activity of sucrose, we incubated P. acnes or S. epidermidis with $2 \%(20 \mathrm{~g} / \mathrm{L})$ sucrose overnight. As shown in Figure S3, incubation of $2 \%$ sucrose did not affect the growth of $P$. acnes or S. epidermidis. The result suggests that a decrease in the number of P. acnes in the co-culture of S. epidermidis and P. acnes with $2 \%$ sucrose (Figure 2A) primarily resulted from $S$. epidermidis sucrose fermentation, not antibacterial property of sucrose. As shown in Figure 3, four SCFAs were produced in the fermented media of S. epidermidis. Results in our previous papers demonstrated that $\mathrm{pH}$ buffering does not influence the antimicrobial activities of SCFAs [15]. Furthermore, SCFAs can passively diffuse through the cell wall of bacteria and kill bacteria by lowering the intracellular $\mathrm{pH}$ [8]. Findings above suggested that the suppression of $P$. acnes growth in a co-culture assay (Figure 2) and by acetic acid (Figure S2) was not simply due to the acidities of SCFAs in media.

By employing an approach of precision programming of the acne microbiome, we use sucrose as a SFI for S. epidermidis in this study. Phage therapy is a precision microbiome approach wherein scientists use bacteriophages specifically targeting pathogens without damaging the commensal bacteria of the host [16]. Although bacteriophages can be highly bacteria-specific, many disadvantages of phage 
therapy have been documented [17]. For example, for the selection of bacteriophages as potential safe antimicrobials, detailed comprehensive characteristics of genome and phenotypic properties may be required, since bacteriophages contain negative features such as lysogeny-associated genes, toxin or enzyme encoding genes [18]. Furthermore, scientists do not yet fully appreciate the safety issue of phage therapy, because many identified genes of bacteriophages express hypothetical and putative proteins with predicted or unknown functions. An additional drawback of phage therapy is that bacteriophages can transfer their genes from one microbe to another. Sucrose-a substance of extremely low acute toxicity—is Generally Recognized As Safe (GRAS) by the US Food and Drug Administration (FDA). With hygroscopic properties, sucrose is commonly used in hair and skin care products to retain moisture. Although sucrose is used here as a SFI for S. epidermidis, future studies will include determining whether sucrose influences the growth and fermentation activity of other commensal bacteria—especially S. aureus—in skin.

Genetic loci responsible for sucrose fermentation of S. epidermidis have not been described. Several enzymes (e.g., permease) regulate the movement of carbohydrates across the bacterial cell wall [19]. We believe that sucrose fermentation, an anaerobic process, also requires many intracellular enzymes that degrade sucrose to SCFAs. Once the key enzyme in charge of sucrose fermentation is determined, a mutant strain of $S$. epidermidis with a deficiency in this enzyme can be created to validate the essential role of fermentation in the suppression of P. acnes growth. Low levels of SCFAs in the human bloodstream were detected, ranging from 3 to $7 \mu \mathrm{M}$ [20]. However, the intestinal microbes in the human colon can locally produce high levels (20-140 mM) of SCFAs [20] that can sufficiently kill pathogens. Although we have not determined how many SCFAs can be locally produced when sucrose is applied to the microenvironment of an acne lesion, it has been documented that SCFAs with short half-lives have apparent difficulty achieving pharmacologic concentrations In Vivo [21]. Several pro-drugs of butyric acid (e.g., pivaloylomethyl butyrate; AN-9) [21] have been developed to achieve effective concentrations of butyric acid. SCFAs act on host cells through at least two mechanisms: inhibition of histone deacetylase (HDAC) and activation of free fatty acid receptors (Ffar1; also known as G-protein coupled receptor 41 (GPR41) and Ffar2; GPR43) [22]. The expression of Ffar1 [23] and Ffar2 [24] in acne lesions is not yet quantified. Several HDAC inhibitors with anti-inflammatory activities are in preclinical and clinical development, including AN-9. Butyric acid is a small molecular weight carboxylate that is a class I HDAC inhibitor [25]. Propionic and butyric acids have been verified as ligands of Ffar1 [26]. Previous studies showed that GW9508 (GlaxoSmithKline)—an arylalkyl derivative of propionic acid-activated the Ffar1 receptor, suppressed chemokine induction in keratinocytes, and attenuated cutaneous immune inflammation [27]. P. acnes can activate toll-like receptor 2 (TLR-2) [28] to stimulate the secretion of IL-6 and IL-8 by follicular keratinocytes, and IL-1 $\beta$, tumor necrosis factor alpha (TNF $\alpha)$, IL-8, and IL-12 by monocytic cells [29]. As shown in Figure 4C, we demonstrated a reduction of MIP-2 cytokine when mouse ear was injected with P. acnes and S. epidermidis in the presence of sucrose. It is possible that SCFAs produced by S. epidermidis attenuate $P$. acnes-induced inflammation via the inhibition of HDACs or the activation of Ffar1 or Ffar2. Our previous studies have demonstrated that a co-drug-butyric acid 2-(2-butyryloxyethoxy) ethyl ester-can release two active butyric acids in mice (data not shown). A co-drug-propionic acid 2-(2-propionyloxy-ethoxy)-ethyl ester-exerts antimicrobial activity [15]. Application of these co-drugs onto acne lesions may conquer the drawbacks of SCFAs with short half-lives for acne treatments.

Our results in Figure 4 show that intradermal injection of $P$. acnes into mouse ears provoked a significant granulomatous response, which was characterized as a lesion of epithelioid macrophages, frequently bounded by a lymphocyte cuff [30]. In the event of severe acne vulgaris, P. acnes could move into the dermal layer once the follicular wall was ruptured [31]. Injection of P. acnes into mouse ears may represent an animal model for the granulomatous type of inflammatory acne vulgaris that follows follicular rupture. P. acnes has been subdivided into at least two types (I and II), and two subtypes, IA and IB [32]. A recent study demonstrated that an acne lesion in humans consists of mixed P. acnes phenotypes [33]. Although we do not know which subtypes of $P$. acnes we isolated from 
acne lesions (Figure S2), the development of SCFA co-drugs that can specifically suppress the growth of acne-associated P. acnes subtypes may be necessary to avoid the disruption of the homeostasis of commensal $P$. acnes subtypes. We also do not know whether all strains of $S$. epidermidis in acne lesions have equal abilities to ferment sucrose. It has been reported that IL-8 was expressed at a higher level in acne lesions than that in healthy skin [34]. As shown in Figure S4A, the level of IL-8 cytokine was significantly reduced when an acne lesion was incubated with $(20 \mathrm{~g} / \mathrm{L})$ sucrose for $24 \mathrm{~h}$. P. acnes bacteria in acne lesions were detected by immunohistochemical staining with a monoclonal antibody against a surface sialidase protein of $P$. acnes. Incubation of sucrose significantly lowered the numbers of $P$. acnes in acne lesions (Figure S4B-F). A clinical study with additional acne lesions to determine the production of SCFAs by sucrose fermentation and the efficacy of sucrose as a SFI for S. epidermidis against $P$. acnes in acne lesions may be required in the future.

Clindamycin and erythromycin are commonly-prescribed topical antibiotics for acne vulgaris [35]. Antibiotic single-agent therapy can result in rapid development of clinically significant antibiotic resistance [36]. Several sugars have been added into antibiotic formula as ingredients. For example, sucrose is often added into amoxicillin for formulation [37]. An adjuvant is a pharmacological or immunological agent that modifies the effect of a drug or vaccine. Formulation of an antibiotic with sucrose which functions as an adjuvant may potentiate the effect of the antibiotic on killing of P. acnes. The development of sucrose as post-antibiotic adjuvant therapy [38] may lower the required dose of antibiotic for the treatment of acne vulgaris, decreasing the risk of generating resistant $P$. acnes and non-specific killing effect of antibiotics on skin commensal bacteria.

\section{Experimental Section}

\subsection{Ethics Statement}

Experiments using mice were performed at National Central University (NCU). The NCU ethics committee specifically approved this study (ID: 104-2320-B-008-003) on August 01, 2015 under an approved Institutional Animal Care and Use Committee (IACUC) protocol.

\subsection{Bacterial Culture}

S. epidermidis bacteria, including ATCC 12228 and non-fermenting S. epidermidis isolated from human skin, were cultured on 3\% tryptic soy broth (TSB) (Sigma, St. Louis, MO, USA) agar plates overnight at $37^{\circ} \mathrm{C}$. P. acnes bacteria (ATCC 6919) were cultured on Brucella broth agar plates, supplemented with $5 \%(v / v)$ vitamin K (Remel, Lenexa, KS, USA) and hemin (Remel) under anaerobic conditions using Gas-Pak (BD Biosciences, San Jose, CA, USA) at $37^{\circ} \mathrm{C}$ with shaking at $200 \mathrm{rpm}$. A single colony was inoculated in 3\% TSB and Reinforced Clostridium Medium (Oxford, UK) and cultured at $37^{\circ} \mathrm{C}$ until the logarithmic growth phase. Bacterial pellets were harvested by centrifugation at $5000 \times g$ for $10 \mathrm{~min}$, washed with PBS, and suspended in PBS.

\subsection{Fermentation of Bacteria}

S. epidermidis and P. acnes bacteria $\left(10^{5} \mathrm{CFU} / \mathrm{mL}\right)$ were incubated in $10 \mathrm{~mL}$ rich media $(10 \mathrm{~g} / \mathrm{L}$ yeast extract (Biokar Diagnostics, Beauvais, France), $3 \mathrm{~g} / \mathrm{L} \mathrm{TSB}, 2.5 \mathrm{~g} / \mathrm{L} \mathrm{K}_{2} \mathrm{HPO}_{4}$, and $1.5 \mathrm{~g} / \mathrm{L} \mathrm{KH}_{2} \mathrm{PO}_{4}$ ) in the absence and presence of $20 \mathrm{~g} / \mathrm{L}$ sucrose under anaerobic conditions at $37^{\circ} \mathrm{C}$ with shaking at $200 \mathrm{rpm}$. The rich media plus $20 \mathrm{~g} / \mathrm{L}$ sucrose without bacteria were included as a control. The $0.002 \%(w / v)$ phenol red (Sigma, St. Louis, MO, USA) in rich media with $20 \mathrm{~g} / \mathrm{L}$ sucrose acted as a fermentation indicator. A color change from red-orange to yellow indicated the occurrence of bacterial fermentation.

\subsection{Co-Culture Assays}

S. epidermidis $\left(10^{6} \mathrm{CFU}\right)$ and P. acnes $\left(10^{6} \mathrm{CFU}\right)$ bacteria were co-incubated in $10 \mathrm{~mL}$ rich media with or without $20 \mathrm{~g} / \mathrm{L}$ sucrose under anaerobic conditions for three days at $37^{\circ} \mathrm{C}$ with shaking 
at $200 \mathrm{rpm}$. After incubation, bacteria were diluted 1:10-1:105 with PBS and then spotted the dilution $(5 \mu \mathrm{L})$ onto $P$. acnes selective agar plates containing rich media and $10 \mu \mathrm{g} / \mathrm{mL}$ of furazolidone (Sigma) [39]. The CFUs of $P$. acnes were counted after incubation at $37^{\circ} \mathrm{C}$ for six days.

\subsection{NMR Analysis}

S. epidermidis (ATCC 12228) $\left(10^{5} \mathrm{CFU} / \mathrm{mL}\right)$ bacteria were incubated in rich media in the presence of ${ }^{13} \mathrm{C}_{12}$-sucrose (20 g/L) (Omicron Biochemicals, Inc., South Bend, USA) for six days. The 1-D NMR spectra of SCFAs were obtained via a $400 \mathrm{MHz}$ JEOL-ECS NMR spectrometer. The 2-D ${ }^{1} \mathrm{H}^{-13} \mathrm{C}$ heteronuclear single quantum correlation (HSQC) spectra of SCFAs were acquired on a Bruker Avance $600 \mathrm{MHz}$ NMR spectrometer with a triple resonance inverse (TCI) cryo-probe. The $2048 \times 256$ complex data points were recorded with 32 scans and a repetition time of $1 \mathrm{~s}$, as previously described in detail [4]. Newly appearing peaks are the intermediate or final products produced by ${ }^{13} \mathrm{C}_{12}$-sucrose fermentation of S. epidermidis.

\subsection{The Sucrose Fermentation of S. epidermidis against P. acnes In Vivo}

The ICR mice (2-3-month-old females; Harlan Labs, Placentia, CA, USA) were anesthetized by isoflurane (Sigma). Five mice per group were used in each experiment. The ears of ICR mice were injected intradermally with P. acnes (ATCC 6919) (10 7 CFU) and S. epidermidis (ATCC 12228) $\left(10^{7} \mathrm{CFU}\right)$ with sucrose $(20 \mathrm{~g} / \mathrm{L}$ in $10 \mu \mathrm{L}$ PBS $)$ or $10 \mu \mathrm{L}$ PBS using an insulin syringe with $29 \mathrm{G} \times 1 / 2$ inches (BD Biosciences, San Jose, CA, USA). After three days, ear thickness was measured with an electronic digital caliper (Mitutoyo, Kanagawa, Japan). Ears were excised, weighed, and homogenized for cytokine detection and bacterial counts. The total protein concentration was measured by a Pierce BCA Protein Assay Kit (Thermo Scientific, Waltham, MA, USA).

\subsection{Bacterial Loads in Mouse Ears}

After excising mouse ears, tissue homogenates were made by a tissue grinder in $200 \mu \mathrm{L}$ of sterile PBS. CFUs of P. acnes in ear homogenates were enumerated by plating serial dilutions $\left(1: 10-1: 10^{5}\right)$ of homogenates on P. acnes selective agar plates containing rich media and $10 \mu \mathrm{g} / \mathrm{mL}$ of furazolidone (Sigma). After that, plates were incubated for three days at $37^{\circ} \mathrm{C}$ under anaerobic conditions using Gas-Pak. A biosafety level 2 (BSL-2) facility was used for conduction of mouse experiments in accordance with institutional guidelines for animal experiments.

\subsection{ELISA}

The MIP-2 in the supernatants of ear homogenates was quantified by an ELISA kit, as directed by the manufacturer (R\&D System. Inc., Minneapolis, MN, USA).

\subsection{Statistics}

Experiments were repeated at least three times with similar results. Statistical significance was determined using Student's unpaired two-tailed $t$-test, as indicated in the legend ${ }^{*} p<0.05,{ }^{* *} p<0.01$, $* * * p<0.001)$.

\section{Conclusions}

Overall, in this study, sucrose was able to specifically intensify the probiotic ability of S. epidermidis, producing SCFAs which can potentially suppress the inflammation and growth of $P$. acnes. The novel acne treatment using sucrose as a SFI for probiotic $S$. epidermidis may benefit the entire community of patients with acne vulgaris, consisting of over 50,000 patients in the US [40].

Supplementary Materials: Supplementary materials can be found at www.mdpi.com/1422-0067/17/11/1870/s1. 
Acknowledgments: This work was supported by NIH grants (5R21AR068005 and 1R41AR064046-01). We thank Ashley Dai and Sherwin Kuo for their participation in the project discussion. We also thank Sunita Keshari for her great effort at editing this manuscript.

Author Contributions: Chun-Ming Huang, Stephen Huang and Richard L. Gallo conceived and designed the experiments; Yanhan Wang, Ming-Shan Kao, and Shinta Marito performed the In Vitro or In Vivo experiments; Jinghua Yu conducted the NMR analysis. Yanhan Wang and Chun-Ming Huang analyzed the data. Chun-Ming Huang wrote the paper.

Conflicts of Interest: The authors declare no conflict of interest.

\begin{tabular}{ll} 
Abbreviations & \\
Ac & acetic acid \\
ATCC & American Type Culture Collection \\
BA & butyric acid \\
CFU & colony-forming unit \\
BSL-2 & biosafety level 2 \\
1-D & one-dimensional \\
2-D & two-dimensional \\
D O & deuterium oxide \\
ELISA & enzyme-linked immunosorbent assay \\
FDA & Food and Drug Administration \\
Ffar1 & free fatty acid receptor 1 \\
Ffar2 & free fatty acid receptor 2 \\
GPR41 & G-protein coupled receptor 41 \\
GRAS & Generally Recognized As Safe \\
HDAC & histone deacetylase \\
HSQC & heteronuclear single quantum coherence \\
IACUC & Institutional Animal Care and Use Committee \\
IL & interleukin \\
MIP-2 & macrophage-inflammatory protein-2 \\
NCU & National Central University \\
NIH & National Institutes of Health \\
NF-S. epidermidis & non-fermentation S. epidermidis \\
NMR & nuclear magnetic resonance \\
NS & non-significant \\
P. acnes & Propionibacterium acnes \\
PBS & phosphate buffered saline \\
SCFA & short-chain fatty acids \\
SD & standard deviation \\
S. epidermidis & Staphylococcus epidermidis \\
SFI & selective fermentation initiator \\
16S rRNA & 16S ribosomal RNA \\
TLR-2 & toll-like receptor 2 \\
TNF $\alpha$ & tumor necrosis factor $\alpha$ \\
& \\
\hline & \\
&
\end{tabular}

\section{References}

1. Grice, E.A.; Segre, J.A. The skin microbiome. Nat. Rev. Microbiol. 2011, 9, 244-253. [CrossRef] [PubMed]

2. Kong, H.H.; Segre, J.A. Skin microbiome: Looking back to move forward. J. Investig. Dermatol. 2012, 132, 933-939. [CrossRef] [PubMed]

3. Scanlan, P.D.; Buckling, A.; Kong, W.; Wild, Y.; Lynch, S.V.; Harrison, F. Gut dysbiosis in cystic fibrosis. J. Cyst. Fibros. 2012, 11, 454-455. [CrossRef] [PubMed]

4. Wang, Y.; Kuo, S.; Shu, M.; Yu, J.; Huang, S.; Dai, A.; Two, A.; Gallo, R.L.; Huang, C.M. Staphylococcus epidermidis in the human skin microbiome mediates fermentation to inhibit the growth of propionibacterium acnes: Implications of probiotics in acne vulgaris. Appl. Microbiol. Biotechnol. 2014, 98, 411-424. [CrossRef] [PubMed]

5. Moss, C.W.; Dowell, V.R., Jr.; Lewis, V.J.; Schekter, M.A. Cultural characteristics and fatty acid composition of corynebacterium acnes. J. Bacteriol. 1967, 94, 1300-1305. [PubMed]

6. Robbins, G.B.; Lewis, K.H. Fermentation of sugar acids by bacteria. J. Bacteriol. 1940, 39, 399-404. [PubMed]

7. Safonova, T.B.; Shcherbakova, N.A.; Afanas'eva, T.I.; Sobolev, V.R. Importance of carbohydrate tests for interspecies differentiation of staphylococci. Zh. Mikrobiol. Epidemiol. Immunobiol. 1978, 9, 98-101. 
8. Shu, M.; Wang, Y.; Yu, J.; Kuo, S.; Coda, A.; Jiang, Y.; Gallo, R.L.; Huang, C.M. Fermentation of propionibacterium acnes, a commensal bacterium in the human skin microbiome, as skin probiotics against methicillin-resistant staphylococcus aureus. PLOS ONE 2013, 8, e55380. [CrossRef] [PubMed]

9. Eady, E.A.; Layton, A.M.; Cove, J.H. A honey trap for the treatment of acne: Manipulating the follicular microenvironment to control propionibacterium acnes. BioMed Res. Int. 2013, 2013. [CrossRef] [PubMed]

10. Levine, R.M.; Rasmussen, J.E. Intralesional corticosteroids in the treatment of nodulocystic acne. Arch. Dermatol. 1983, 119, 480-481. [CrossRef] [PubMed]

11. Layton, A.M.; Dreno, B.; Gollnick, H.P.; Zouboulis, C.C. A review of the european directive for prescribing systemic isotretinoin for acne vulgaris. J. Eur. Acad. Dermatol. Venereol. 2006, 20, 773-776. [CrossRef] [PubMed]

12. Lindh, J.M.; Terenius, O.; Faye, I. 16s rRNA gene-based identification of midgut bacteria from field-caught anopheles gambiae sensu lato and $A$. funestus mosquitoes reveals new species related to known insect symbionts. Appl. Environ. Microbiol. 2005, 71, 7217-7223. [CrossRef] [PubMed]

13. Al-Naama, R.T. Evaluation of inhibitory effect of honey on some bacterial isolates. Iraqi J. Med. Sci. 2009, 7, 67-72.

14. Ballesteros, S.A.; Chirife, J.; Bozzini, J.P. Specific solute effects on staphylococcus aureus cells subjected to reduced water activity. Int. J. Food Microbiol. 1993, 20, 51-66. [CrossRef]

15. Wang, Y.; Dai, A.; Huang, S.; Kuo, S.; Shu, M.; Tapia, C.P.; Yu, J.; Two, A.; Zhang, H.; Gallo, R.L.; et al. Propionic acid and its esterified derivative suppress the growth of methicillin-resistant Staphylococcus aureus USA300. Benef. Microbes 2014, 5, 161-168. [CrossRef] [PubMed]

16. Pincus, N.B.; Reckhow, J.D.; Saleem, D.; Jammeh, M.L.; Datta, S.K.; Myles, I.A. Strain specific phage treatment for staphylococcus aureus infection is influenced by host immunity and site of infection. PLoS ONE 2015, 10, e0124280. [CrossRef] [PubMed]

17. Haq, I.U.; Chaudhry, W.N.; Akhtar, M.N.; Andleeb, S.; Qadri, I. Bacteriophages and their implications on future biotechnology: A review. Virol. J. 2012, 9. [CrossRef] [PubMed]

18. Drulis-Kawa, Z.; Majkowska-Skrobek, G.; Maciejewska, B.; Delattre, A.S.; Lavigne, R. Learning from bacteriophages-Advantages and limitations of phage and phage-encoded protein applications. Curr. Protein Pept. Sci. 2012, 13, 699-722. [CrossRef] [PubMed]

19. Jahreis, K.; Pimentel-Schmitt, E.F.; Brückner, R.; Titgemeyer, F. Ins and outs of glucose transport systems in eubacteria. FEMS Microbiol. Rev. 2008, 32, 891-907. [CrossRef] [PubMed]

20. Garland, S.H. Short chain fatty acids may elicit an innate immune response from preadipocytes: A potential link between bacterial infection and inflammatory diseases. Med. Hypotheses 2011, 76, 881-883. [CrossRef] [PubMed]

21. Hobdy, E.; Murren, J. AN-9 (Titan). Curr. Opin. Investig. Drugs 2004, 5, 628-634. [PubMed]

22. Vinolo, M.A.; Rodrigues, H.G.; Nachbar, R.T.; Curi, R. Regulation of inflammation by short chain fatty acids. Nutrients 2011, 3, 858-876. [CrossRef] [PubMed]

23. Ulven, T. Short-chain free fatty acid receptors FFA2/GPR43 and FFA3/GPR41 as new potential therapeutic targets. Front. Endocrinol. 2012, 3. [CrossRef] [PubMed]

24. Gottlicher, M. Valproic acid: An old drug newly discovered as inhibitor of histone deacetylases. Ann. Hematol. 2004, 83, S91-S92. [PubMed]

25. Silva, F.; Serpa, J.; Domingues, G.; Silva, G.; Almeida, A.; Félix, A. Cell death induced by HDACS inhibitors in ovarian cancer cell lines (serous and clear cells carcinomas)—Role of NOTCH, TP53 and FN1. BMC Proc. 2010, 4. [CrossRef]

26. Park, J.S.; Lee, E.J.; Lee, J.C.; Kim, W.K.; Kim, H.S. Anti-inflammatory effects of short chain fatty acids in IFN- $\gamma$-stimulated RAW 264.7 murine macrophage cells: Involvement of Nf-kB and ERK signaling pathways. Int. Immunopharmacol. 2007, 7, 70-77. [CrossRef] [PubMed]

27. Hara, T.; Hirasawa, A.; Ichimura, A.; Kimura, I.; Tsujimoto, G. Free fatty acid receptors FFAR1 and GPR120 as novel therapeutic targets for metabolic disorders. J. Pharm. Sci. 2011, 100, 3594-3601. [CrossRef] [PubMed]

28. Kim, J. Review of the innate immune response in acne vulgaris: Activation of toll-like receptor 2 in acne triggers inflammatory cytokine responses. Dermatology 2005, 211, 193-198. [CrossRef] [PubMed] 
29. Mastrofrancesco, A.; Kokot, A.; Eberle, A.; Gibbons, N.C.; Schallreuter, K.U.; Strozyk, E.; Picardo, M.; Zouboulis, C.C.; Luger, T.A.; Bohm, M. KdPT, a tripeptide derivative of $\alpha$-melanocyte-stimulating hormone, suppresses IL-1 $\beta$-mediated cytokine expression and signaling in human sebocytes. J. Immunol. 2010, 185, 1903-1911. [CrossRef] [PubMed]

30. Liu, P.F.; Nakatsuji, T.; Zhu, W.; Gallo, R.L.; Huang, C.M. Passive immunoprotection targeting a secreted CAMP factor of Propionibacterium acnes as a novel immunotherapeutic for acne vulgaris. Vaccine 2011, 29, 3230-3238. [CrossRef] [PubMed]

31. Kligman, A.M. An overview of acne. J. Investig. Dermatol. 1974, 62, 268-287. [CrossRef] [PubMed]

32. Valanne, S.; McDowell, A.; Ramage, G.; Tunney, M.M.; Einarsson, G.G.; O’Hagan, S.; Wisdom, G.B.; Fairley, D.; Bhatia, A.; Maisonneuve, J.F.; et al. CAMP factor homologues in Propionibacterium acnes: A new protein family differentially expressed by types I and II. Microbiology 2005, 151, 1369-1379. [CrossRef] [PubMed]

33. Jahns, A.C.; Lundskog, B.; Ganceviciene, R.; Palmer, R.H.; Golovleva, I.; Zouboulis, C.C.; McDowell, A.; Patrick, S.; Alexeyev, O.A. An increased incidence of propionibacterium acnes biofilms in acne vulgaris: A case-control study. Br. J. Dermatol. 2012, 167, 50-58. [CrossRef] [PubMed]

34. Kelhälä, H.L.; Palatsi, R.; Fyhrquist, N.; Lehtimäki, S.; Väyrynen, J.P.; Kallioinen, M.; Kubin, M.E.; Greco, D.; Tasanen, K.; Alenius, H.; et al. IL-17/Th17 pathway is activated in acne lesions. PLoS ONE 2014, 9, e105238. [CrossRef] [PubMed]

35. Del Rosso, J.Q.; Schmidt, N.F. A review of the anti-inflammatory properties of clindamycin in the treatment of acne vulgaris. Cutis 2010, 85, 15-24. [PubMed]

36. Zavascki, A.P.; Bulitta, J.B.; Landersdorfer, C.B. Combination therapy for carbapenem-resistant Gram-negative bacteria. Expert Rev. Anti Infect. Ther. 2013, 11, 1333-1353. [CrossRef] [PubMed]

37. Soares, D.N.; Antonio, A.G.; Iorio, N.L.; Pierro, V.S.; dos Santos, K.R.; Maia, L.C. Does the presence of sucrose in pediatric antibiotics influence the enamel mineral loss and the Streptococcus mutans counts in dental biofilm? Braz. Dent. J. 2015, 26, 249-257. [CrossRef] [PubMed]

38. Hurley, M.N.; Forrester, D.L.; Smyth, A.R. Antibiotic adjuvant therapy for pulmonary infection in cystic fibrosis. Cochrane Database Syst. Rev. 2013. [CrossRef]

39. Marino, C.; Stoughton, R.B. Clinical use of a selective culture medium for wild and antibiotic-resistant propionibacterium acnes. J. Am. Acad. Dermatol. 1982, 6, 902-908. [CrossRef]

40. Fried, R.G.; Wechsler, A. Psychological problems in the acne patient. Dermatol. Ther. 2006, 19, $237-240$. [CrossRef] [PubMed] 